\title{
Case Report: Association between Breast Cancer and Silicone Breast Prosthesis
}

\section{Vila Garcia $\mathrm{E}^{1 *}$, Tamayo Carbon $\mathrm{AM}^{2}$, Ortiz Roque J ${ }^{3}$, Ramon Concepcion $\mathrm{I}^{4}$, Estrada Napoles $\mathrm{Y}^{5}$ and Estevez Medina $\mathrm{K}^{6}$}

${ }^{1}$ Specialist I degree in General Surgery, Surgical Hospital Hermanos Ameijeiras, Cuba

${ }^{2}$ Specialists of II degree in Plastic Surgery and Caumatology, Surgical Hospital Hermanos Ameijeiras, Cuba

${ }^{3}$ Specialist I degree in Radiology, Surgical Hospital Hermanos Ameijeiras, Cuba

${ }^{4}$ Specialist I degree in Clinical Oncology, Surgical Hospital Hermanos Ameijeiras, Cuba

${ }^{5}$ Specialist I degree in Pathological Anatomy. Surgical Hospital Hermanos Ameijeiras, Cuba

${ }^{6} 2$ nd Year Resident of Plastic Surgery and Caumatology, Surgical Hospital Hermanos Ameijeiras, Cuba

*Corresponding author: Eyleen Vila Garcia, Assistant Professor, Services of General Surgery and Plastic Surgery, Surgical Hospital Hermanos Ameijeiras, Havana City, Cuba, Tel: 53232327; Email: hielenvg@infomed.sld.cu

\section{Abstract}

The health risks caused by silicone breast implants have been debated since the last century, demonstrating their safety and effectiveness. There is no known direct association with breast cancer, although isolated cases with anaplastic giant cell lymphoma are reported. We describe a patient with infiltrating ductal carcinoma of the breast, who underwent augmentation mastoplasty with breast implants 10 years earlier. We analyze a case of 64-year-old female patient with a history of augmentation mastoplasty is presented, who consults for retraction of the skin of the breast. On physical examination, there is retraction of the skin and an irregular $2.5 \mathrm{~cm}$ nodule in the super external quadrant of the left breast. Breast ultrasound, mammography, and simple chest CT were performed, reporting left breast cancer with metastases to the left axillaries nodes and intracapsular rupture of the left breast prosthesis. Ultrasound-guided fine needle aspiration cytology of the mammary nodule was performed, reporting positive for neoplastic cells. The intraoperative finding was a $3 \mathrm{~cm}$ tumor in the upper external quadrant of the left breast and the contained intracapsular rupture of the left breast prosthesis, performing lumpectomy with left axillary emptying and removal of both prostheses. The histological result reports infiltrating ductal carcinoma with metastasic axillaries lymphadenopathy and gigantocellular foreign body granulomatous reaction. It can be affirmed that patients with breast implants have a lower incidence of breast cancer than the general population.

Keywords: Breast Implants; Breast Cancer

\section{Introduction}

Knowledge of the anatomy, embryology of the breast, armpit and pectoral muscles is essential to perform the different surgical procedures in Mastology. Also to identify the most frequent sites affected by Breast Cancer (BC), local recurrence and plan oncospecific treatments. Silicone breast implants are mainly used for cosmetic augmentation mastoplasty and less frequently in BC oncoplastic surgery after a lumpectomy or mastectomy [1].

The main indications for breast implants are: aesthetic (amastia, hypoplasia, hypotrophy, and breast ptosis), reconstructive for congenital or acquired benign 


\section{International Journal of Transplantation \& Plastic Surgery}

malformations (anisomastias, Poland syndrome, tuberous breast), and BC oncoplastic surgery. Other less common indications are unilateral adolescent hypertrophy, fibrocystic disease and prophylactic mastectomy due to high risk of breast cancer [2-3].

The most used prosthetic materials for augmentation mastoplasty and tissue expansion are silicone implants, saline or silicone gel filled [4-6].

Silicone breast implants have been used in the United States (US) and Europe for many years without evaluation of toxicity and its complications. Initially, documentation of the composition of the implant, or of the person operated on, was not required and there was no exhaustive systematic surveillance of the positive or negative long-term consequences. In the $90 \mathrm{~s}$, concern about silicone for health arose in the USA and studies were carried out that motivated the US Food and Drug Administration (FDA) to include all breast implants in the category of category III (which require strict safety standards and effectiveness) [4].

The health risks caused by silicone breast implants have been widely debated since the last century. Numerous epidemiological studies demonstrate the safety of silicone breast implants and that they do not increase the incidence of BC. However, the association of breast implants with Anaplastic Large-Cell Lymphoma is known. (ALCL). This is a cancer of the hemolymphopoietic system that does not involve the mammary parenchyma and in Cuba there is no registered case [1,3,4,7-10].

$\mathrm{BC}$ is the rampant growth of malignant cells in breast tissue. There are two main types:

Ductal carcinoma: It is the most frequent and begins in the ducts that carry milk from the breast to the nipple.

Lobular carcinoma: It begins in the lobules that are those that produce breast milk [11].
The cause remains unknown despite the discovery of multiple risk factors. Multifactorial etiology is considered, but implants are not mentioned in the literature as a risk factor. Among the main risk factors for $\mathrm{BC}$ are: advanced age, early menarche, nulliparity or age of first delivery over 30 years, family history of $\mathrm{BC}$, hormone consumption (oral estrogen and progesterone contraceptives), alcohol consumption, hereditary genetic mutations (BRCA1 and BRCA2) between 5 and $10 \%$ and previous exposure to ionizing radiation[11,12].

$\mathrm{BC}$ is the most common female sex and the leading cause of death from cancer in all age groups. In recent years the incidence of $\mathrm{BC}$ is increasing in younger women, being around $5 \%$ in women younger than 40 years. In these patients, the prognosis is worse due to presenting a more aggressive histology and a higher recurrence rate [13].

It is estimated that 1 in 14 women will be affected by a $\mathrm{BC}$ throughout their lives. In the future year 2030 there will be 52077 cancer patients in Cuba, and more than 1.6 million women in the world will die from $\mathrm{BC}$ [10]. This gives us the not only medical but social importance of this disease. For this reason, the investigation of the association between breast implants and BC is proposed.

\section{Case Report}

64-year-old female patient with a history of having undergone an augmentation Mastoplasty with the placement of textured silicone breast implants and retropectoral implants periareolar approach 10 years ago, without other personal pathological history, who came to the clinic for presenting skin retraction of the left breast. He denies a family history of breast, prostate, and ovarian cancer. He denies allergies and medication consumption. On physical examination, retraction of $1 \mathrm{~cm}$ of the skin is observed and an irregular $2.5 \mathrm{~cm}$ nodule is palpated in the super external quadrant of the left breast without other alterations (Figure 1).
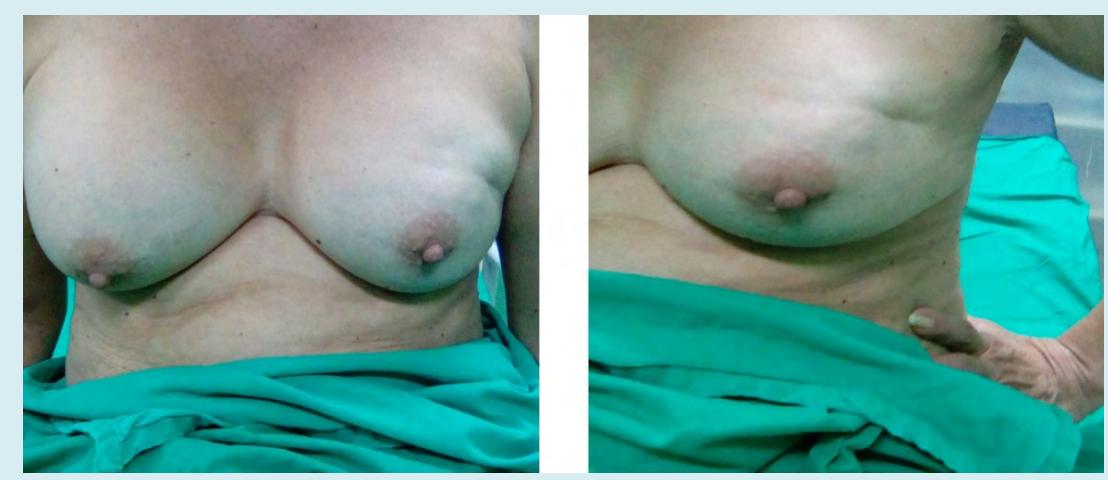

Figure 1: Patient with a breast prosthesis operated 10 years ago who presented a $2.5 \mathrm{~cm}$ tumor in the upper external quadrant of the left breast that retracts the skin. A. Front view. B. Oblique view. 


\section{International Journal of Transplantation \& Plastic Surgery}

Ultrasound imaging studies of the breasts, mammography by special Eklund technique (Figure 2), and simple chest CT (Figure 3) were performed, reporting left breast cancer with metastasis to the left axillaries nodes and intracapsular rupture of the left breast prosthesis.
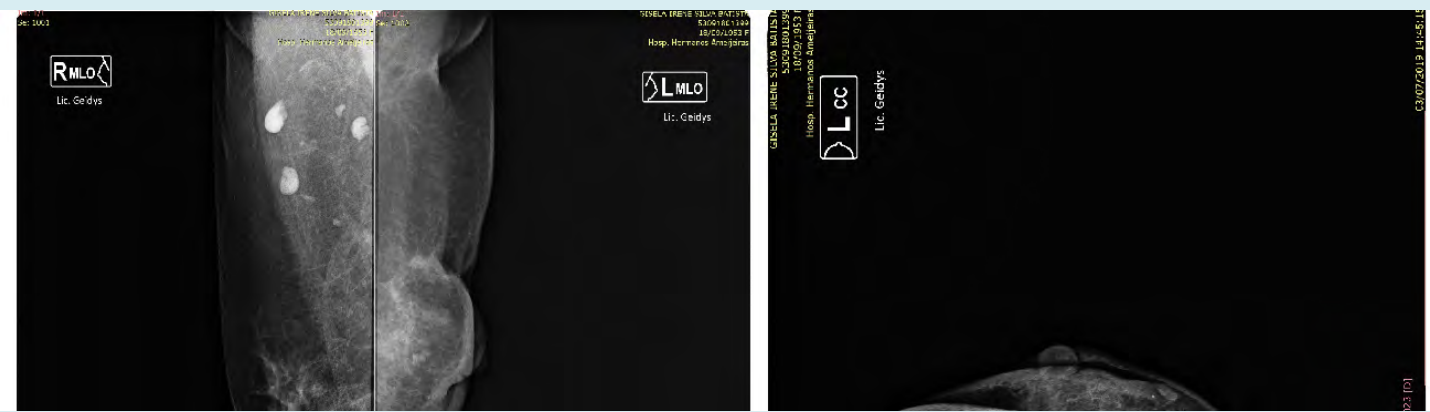

Figure 2: Mammography using the Eklund technique. A: Oblique mediolateral view of the right breast. B: Mediolateral oblique view of the left breast. C: Craniocaudal view of the left breast.

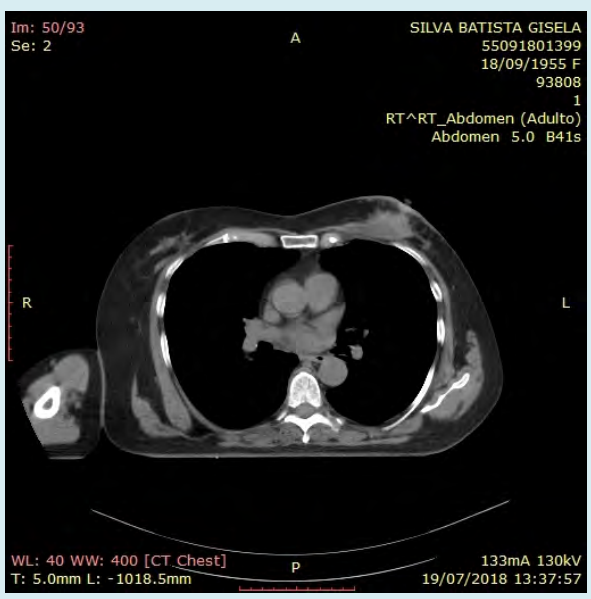

Figure 3: Simple computed tomography of the chest showing a tumor in the upper external quadrant of the left breast with metastatic left axillaries lymphadenopathy and intracapsular rupture of the left breast prosthesis.
Ultrasound-guided fine needle aspiration cytology of the breast node was performed, reporting positive for neoplastic cells.

With all these elements, the patient is evaluated in the Central Breast Cancer Consultation by the multidisciplinary group of Mastology (Composed of the specialties of Clinical Oncology, General Surgery, Plastic Surgery, Radiology, Pathological Anatomy, Nuclear Medicine, Physiotherapy and Psychology) and it was decided to start with surgical treatment and continues with oncospecific adjuvant treatment.

In surgery, a 3-cm losange incision was made in the super external quadrant of the left breast, a 3 -cm tumor was resected, and intracapsular rupture of the prosthesis covered by the retropectoral periprosthetic capsule was confirmed with no content leakage.
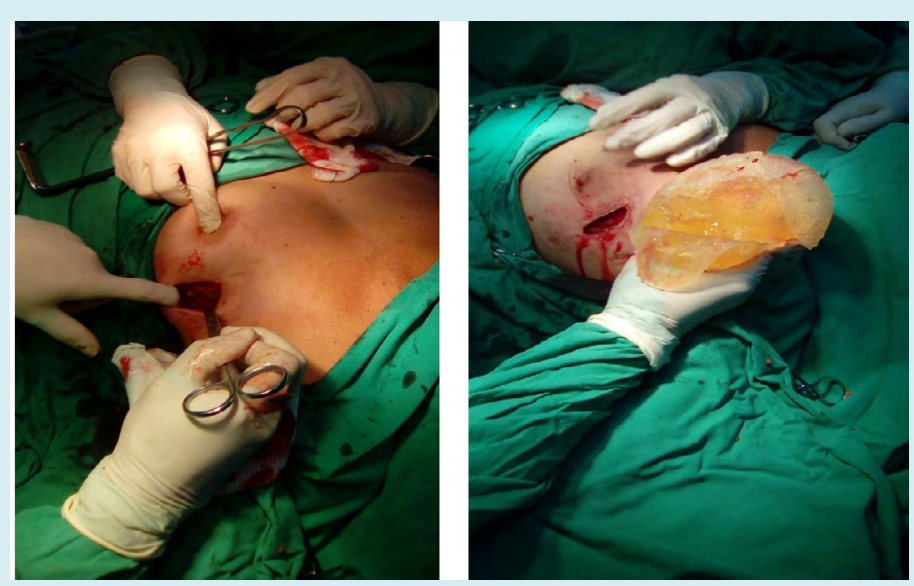

Figure 4: Intraoperative findings. A: Arc-shaped incision in the super external quadrant of the left breast through which oncological surgery was performed. B: Left breast prosthesis removed and with the broken silicone capsule on its anterior face. 


\section{International Journal of Transplantation \& Plastic Surgery}

Lumpectomy and freeze biopsy were performed, reporting infiltrating ductal carcinoma $3 \times 2 \mathrm{~cm}$ in diameter with tumor-free surgical section edges. The left axillary was emptied and both prostheses were removed by a single $3 \mathrm{~cm}$ long arcuate incision in the super external quadrant of the left breast and another same size incision in the submammary sulcus of the right breast. No replacement of the prostheses is made at the request of the patient (Figure 4).

The histological result of the paraffin biopsy reports $3 \mathrm{x}$ $2 \mathrm{~cm}$ infiltrating ductal carcinoma with tumor-free surgical section edges. Metastasis in 6 lymph nodes of 15 dissected (6/15). Histological grade II, nuclear grade III, solid pattern, permeation of lymphatic vessels, high mitotic index. There is presence of a gigantocellular granulomatous reaction to a foreign body type.

IHQ: RE: $90 \%$ intense positive.

RP: $20 \%$ intense positive.

Her2: negative 0.

Ki67: positive in more than 20\% (40\%) (Figure 5).

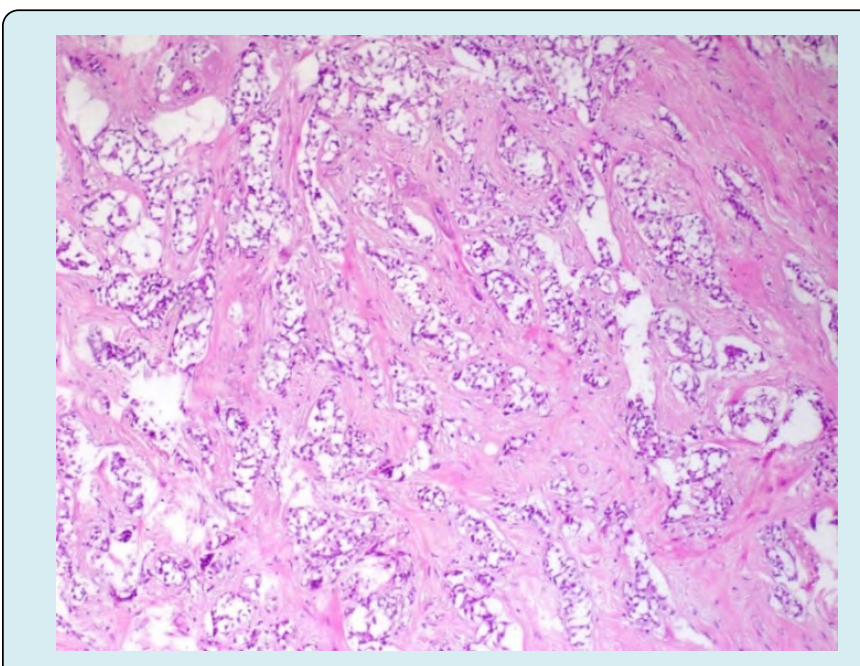

Figure 5: Paraffin biopsy of infiltrating ductal carcinoma.

The patient evolved without complications and in the immediate postoperative period she was discharged complying with the measures of the ERAS protocols with satisfactory aesthetic and oncological results.

An outpatient surgery appointment was scheduled 4 days after surgery to heal the wounds, remove continuous left axillary aspiration drainage, and explain post-operative care and exercises on the left arm.

The surgery consultation continues 7 days later and 15 days postoperatively the stitches are removed and the patient is discharged with remission for the Oncology and Physiotherapy consultation.
An oncospecific adjuvant treatment with sequential polychemotherapy $(4 \mathrm{AC}+4 \mathrm{Tx})$, radiation therapy to the breast and armpit plus hormone therapy with Letrozole 2.5 mg 1 tablet daily for 5 years is indicated by Oncology.

In addition, in Physiotherapy consultation, the exercises to elevate the operated arm and all the general care of the arm and the left hand are explained in detail. Currently, she has been operated for 2 years and has not presented complications or relapse of her disease.

\section{Discussion}

Silicone has been used worldwide since the 1980s for implants throughout the body (heart valves and other cardiovascular prostheses; catheters; dentistry; gastrointestinal tract; ophthalmology; ears, nose, throat, and respiratory tract; urogenital tract, including prostheses). Penis; as a prosthesis or prosthetic ingredient for a large part of the skeletal system; as a tissue dilator, as a cosmetic agent for the treatment of scars and wrinkles) [4]. This article is limited to cohesive silicone gel implants of the breast and its relationship with the $\mathrm{BC}$.

There are several types of breast implants, most have a silicone capsule and what varies is the filling material used in the implants (silicone gel, saline, and soybean oil, hydrogel, mixed in double lumen) and the surface that can be smooth, textured or microtextured [4-6].

In the Pubmed / Medline literature there are 20 publications related to breast implants and BC. Of these, 9 are relevant and a single study is appropriately designed to rule out the association between breast implants and $\mathrm{BC}{ }^{1}$ Several studies and a meta-analysis show that the risk of BC for women with breast implants is lower than for the general population, suggesting that they have a long-term protective effect against BC. In addition, there is no statistically significant increase with the time of exposure to the implant (19-21 years) or the type of implant [1,3,4,7-9].

Epidemiological studies of the health impact of silicone implants focus on the relationship with cancer and connective tissue diseases. The literature confirms that there is no association of breast implants with $\mathrm{BC}$, although isolated cases of primary breast lymphoma are reported [14-20]. However, the relationship with neurological diseases, connective tissue diseases of autoimmune cause (scleroderma) is reported. Lupus erythematosus, rheumatoid arthritis, Sjogren's syndrome, dermatomyositis, fibromyalgia, and chronic fatigue syndrome) and there are no risks to infants or interfering with lactation [4]. 


\section{International Journal of Transplantation \& Plastic Surgery}

Silicone breast implants have a limited life of 10 years, which implies a second surgery for their replacement and exhaustive information to patients from the first surgery. This is because silicone stimulates the chronic inflammatory response with the production of autoantibodies, which could influence the risk of the development of primary breast ALK1 negative ALCL in silicone implant recipients. [1,4,14-20]

Surgical techniques for breast implant placement are perfected on a daily basis looking for the least amount of visible scars and a perfect augmentation mastoplasty technique. The route of insertion of breast prostheses can be local (Cronin and Gerow, 1974; Arion, 1965), regional (Hoehler, 1973) or remote (Planas, 1976; Barrett, 1980). Local insertion is performed through submammary incisions, preferably periareolar or through a previous scar. The regional insertion is carried out by axillary route and the remote insertion is made through an incision far from the breast, armpit or chest. Remote insertion is performed by abdominoplasty incision (Cronin and Gerow, 1974; Arion, 1965) [21]. The evolution of these surgical techniques is directed towards better visualization, better breast and body shaping, smaller scars and less morbidity.

In 1993, Johnson and Christ took advantage of introducing deflated inflatable saline prostheses through a small incision and introduced the surgical technique of transumbilical endoscopic additive breastplasty by placing the subglandular prosthesis. Furthermore, at that time only saline prostheses were available in the USA and the discovery that textured saline prostheses produced a lower incidence of capsular contracture than silicone prostheses made this technique very popular. This is explained by the fact that textured implants can disorganize capsular fibrotic reactions and thus decrease capsular contracture [3,21]. This technique is safe, repeatable and effective for patients, but it is recommended to have at least one additional reserve prosthesis in surgery.

It is noteworthy that our hospital is the National Reference Center for Mastology in Cuba, and an average of 200 patients with $\mathrm{BC}$ are operated each year. In the last 5 years this was the only patient operated on by BC who had previously placed breast implants.

The presenting patient underwent surgery 10 years ago for augmentation mastoplasty with textured silicone implants with local insertion by periareolar incision and retropectoral placement. The literature suggests that the textured prosthesis produces less capsular contracture and the placement of the subglandular plane reduces the risk of BC. However, Augmentation Mastoplasty is a surgery with a high demand in developed countries, but the rupture rate of the prostheses is unknown, although it is stated that the rupture does not increase the risk of $\mathrm{BC}$ but rather that of capsular contracture $[3,12,22]$. In our setting, the submuscular placement plane is preferred for implants, which represents an advantage in this case from an aesthetic and oncological point of view because it prevented early secondary ptosis and extravasation of the extracapsular content of the broken prosthesis.

The intraoperative finding was a $3 \mathrm{~cm}$ tumor in the super external quadrant of the left breast and contained rupture of the left breast prosthesis. Lumpectomy, left axillary emptying and removal of both prostheses at the request of the patient, with acceptable cosmetic results. Other surgical treatment options were the Skin Sparing Left Modified Radical Mastectomy with replacement of both prostheses, but the patient refused to change the implants. In our center, in addition to complying with all surgical and oncological principles, the patient's desire is an absolute criterion for defining conservative or radical surgery in $\mathrm{BC}$ [23].

\section{Comments}

The reported case had as main symptom the retraction of the skin of the breast, which is a warning sign for women. Although the patient's priority is to live, oncology and surgery can always be combined to improve body image. The surgeon must focus on the challenge of complying with all the principles of surgical oncology to obtain a good cosmetic result, increase survival and quality of life for patients.

\section{Conclusions}

Women who undergo Augmentation Mastoplasty with silicone implants have a lower risk of developing BC in the future than the general population, since the implants have a long-term protective effect against BC. Compliance with the BC Early Detection Program and the attention of the multidisciplinary team of Mastology, allows for early diagnosis and oncospecific treatment to increase the survival and quality of life of patients.

\section{Recommendations}

Apply Breast Cancer Early Detection Program for all women. Carry out long-term multicenter studies that allow us to reach conclusions and change paradigms.

\section{Study Limitations}

At the time of diagnosis of this patient ( 2 years ago) we did not have MRI equipment, so this preoperative imaging study is not performed. However, a simple chest CT scan allowed the correct preoperative diagnosis to be made. 


\section{International Journal of Transplantation \& Plastic Surgery}

The needles for performing trucut biopsies are insufficient, therefore they are reserved for patients who are evaluated at the Multidisciplinary Breast Cancer Consultation and require histological diagnosis to start onco specific neoadjuvant treatment.

However, the preoperative diagnosis was correct with the diagnostic means used. However, if MRI and trucut biopsy had been performed, the planned treatment was the same.

\section{References}

1. Eline C Noels, Oren Lapid, Jan H N Lindeman, Esther Bastiaannet (2015) Breast implants and the risk of Breast Cancer: A Meta-Analysis of Cohort Studies. Aesthet Surg J 35(1): 55-62.

2. Sabiston (2017) Surgery Treaty .Section VII: Breast. $19^{\text {th }}$ (Edn.).

3. Bondurant S, Ernster V, Herdman R (1999) Institute of Medicine (US) Committee on the Safety of Silicone Breast Implants; editors. Safety of Silicone Breast Implants. Washington (DC): National Academies Press (US).

4. (2000) Health risks caused by silicone implants in general, with special attention to breast implants. Stoa report / European Parliament. Health Technology Assessment Report No. 23.

5. Tamayo Carbon A, Castellanos Prada A, Aveiro Robalo $\mathrm{T}$ (2018) Iatrogenic alogenosis and rheumatic diseases. Revista Cubana de Reumatologia 20(2): e18

6. Tamayo Carbon A, Estevez del Toro M, Alvarado Salas R, Chong Lopez A (2019) Autoimmune inflammatory syndrome induced by adjuvant after administration of a modeling agent with fibromyalgia symptoms. Rev Colomb Reumatol 26(2).

7. Sai Yi Pan, Eric Lavigne, Eric J Holowaty, Paul J Villeneuve, Lin Xie, et al. (2012) Canadian breast implant cohort: Extended follow-up of cancer incidence. Int J Cancer 131(7): E1148-E1157

8. Scott L Spear, Diane K Murphy, Allergan Silicone Breast Implant US Core Clinical Study Group (2014) Natrelle Round Silicone Breast Implants: Core Study Results at 10 Year. Plast Reconstr Surg 133(6): 1354-1361.

9. Brinton LA, Brown SL (1997) Breast implants and cancer. J Natl Cancer Inst 89(18): 1341-1349.

10. Statistical Yearbook of Health (2018) Ministry of Public Health. Directorate of Medical Records and Health Statistics. Havana.
11. Tulio Roa T, Bohorquez C (2014) Surgical anatomy of the breast. In: Berrocal Revueltas M. The four seasons of the breasts. Evaluation, design and application of surgical procedures, Medical Printing, Bogota, pp: 35-48.

12. American Cancer Society (2014) Breast cancer.

13. Luciano Stover AC, Montejo Viamontes NO, Loys Fernandez JL, Vila Garcia E (2018) Outcome of surgical treatment of breast cancer in women under 40 years of age. Rev Cub Cir 57 (2): 1-11.

14. Roberto N. Miranda, Tariq N Aladily, H Miles Prince, Shamanna RK, Daphne de Jong, et al. (2014) Breast implant-Asociated Anaplastic Large-Cell Lymphoma: Long-Term Follow-Up of 60 Patients. J Clin Oncol 32(2): 114-120.

15. Yi Shan Lee, Armando Filie, Diane Arthur, Antonio T Fojo, Elaine S Jaffe (2015) Breast-implant-associated anaplastic large cell lymphoma in a patient with $\mathrm{Li}$ Fraumeni syndrome. Histopathology 7(6): 925-927.

16. Eva V. George John Pharm, Courtney Houston, Semar AlQuran, Grey Brian, et al. (2013) Breast implant-asociated ALK- negative anaplastic large-cell lymphoma: a case report and discussion of possible pathogenesis. Int J Clin Exp Pathol 6(8): 1631-1642

17. Shiyong Li, Andrew K Lee (2010) Silicone implant and primary breast ALK1- negative anaplastic large-cell lymphoma, fact or fiction? Int J Clin Exp Pathol 3(1): 117127

18. Montgomery Goecker C, Fuda F, Krueger JE, Chen W (2015) Immunophenotypic Characteristics of Breast Implant-Associated Anaplastic Large-Cell Lymphoma by Flow Cytometry. Cytometry B Clin Cytom 88(5): 291293.

19. Mustata L, Collins G, Ilie D, Parulekar V, Adams R, et al. (2019) Case Report of a Node Positive Breast Implant Associated - Anaplastic Large Cell Lymphoma. Int J Transplant \& Plastic Surg 3(2): 000138.

20. Tamayo Carbon A, Cairos Baez J, Chong Lopez A, Alvarado Salas R, Cedillo Lopez R (2019) Post-mastoplasty of Augmentation Late Seroma with Implant. Acta Medica 20(1).

21. F. Ferrari, I. Pintanguy (1999) Plastic surgery. Preoperative strategies. Surgical technique. $1^{\text {st }}$ (Edn.), Chapter 27: Transumbilical Endoscopic Additive Mamaplasty.

22. Collado Mesa, Monica Yepes, Purvi Doshi, Saleem A Umar, J Net (2013)Contralateral Intramammary Silicone 


\section{International Journal of Transplantation \& Plastic Surgery}

Lymphadenitis in a Patient with an Intact Standard Dual Lumen Breast Implant in the Opposite Reconstructed Breast. J Radiol Case Rep 7(11): 24-31.

23. Soriano Garcia JL, Montejo Viamontes N, Loys Fernandez
JL, Lima Perez M, Castillo Carrillo C, et al. (2012) Tumor functional unit. T-I. $4^{\text {th }}$ (Edn.), In: Manual of medical practices, Ed Editorial Medical Sciences. Havana, pp: 2163.

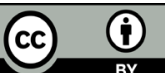

